\title{
Diseminación leptomeníngea de un astrocitoma pilocítico cervical en el adulto: publicación de un caso y revisión de la literatura
}

I. Jusué-Torres; L. Alcázar-Vaquerizo; J.C. Gómez-Angulo; R. Navarro-Torres; R. López-Serrano y N. GarcíaMiralles*

Servicios de Neurocirugía y de Anatomía Patológica*. Hospital Universitario de Getafe.

Resumen

Introducción. La localización menos frecuente de los astrocitomas pilocíticos es la intramedular. Los gliomas representan hasta el $\mathbf{2 4 - 3 0 \%}$ de los tumores intramedulares en el adulto y son los segundos, sólo tras los ependimomas. La diseminación leptomeníngea a través del líquido cefalorraquídeo es inusual y sucede predominantemente en meduloblastomas, ependimoblastomas, neuroblastomas centrales, ependimomas, tumores de células germinales y gliomas de alto grado. La mayoría de las metástasis secundarias a gliomas medulares publicadas proceden de astrocitomas anaplásicos o glioblastomas multiformes y relativamente pocas de gliomas de bajo grado. La incidencia de diseminación leptomeníngea de gliomas de bajo grado es rara. Publicamos una rara diseminación a las leptomeninges cerebrales de un astrocitoma pilocítico intramedular en el adulto.

Caso clínico. Describimos un varón de 51 años con masa intramedular recurrente a nivel de $\mathrm{C} 5-\mathrm{C} 7$ intervenido en 4 ocasiones con todos los informes de anatomía patológica describiendo la lesión como astrocitoma pilocítico. 15 años después del diagnóstico desarrolló alucinaciones visuales y deterioro del nivel de conciencia con escala de coma de Glasgow de 13/15. La resonancia magnética mostró importante realce de las leptomeninges y paquimeninges craneales y medulares con intensa afectación de las cisternas basales, espacio subaracnoideo $y$ ambos hemisferios cerebrales sugerente de diseminación leptomeníngea de la masa medular. El paciente falleció a los 3 días.

Conclusión. Este fenómeno es raro y cuando acontece no suele cambiar el comportamiento del tumor primario. En nuestro caso la agresividad se podría justificar o por una malignización del tumor primario no evidenciada debido a la resección parcial de las últimas cirugías o bien a que el tumor en realidad fuese un tumor pilomixoide monomorfo.

Recibido: 9-10-10. Aceptado: 26-01-11
PALABRAS CLAVE. Tumor intramedular. Diseminación leptomeníngea. Glioma de bajo grado. Astrocitoma pilocítico.

Leptomeningeal spread of an intramedullary cervical pilocytic astrocytoma: case report and literature review

Summary

Background. The rarest location of pilocytic astrocytoma is intramedullary. Gliomas represent up to $\mathbf{2 4}$ $\mathbf{- 3 0 \%}$ of intramedullary tumors in adulthood and are second only after ependymomas. Leptomeningeal dissemination through cerebrospinal fluid is unusual and occurs predominantly in medulloblastomas, ependymoblastomas, central neuroblastomas, ependymomas, germ cell tumors and high-grade gliomas. The majority of spinal cord gliomas reporting metastasis were anaplastic astrocytomas or glioblastomas multiforme and relatively few were low-grade gliomas. The incidence of leptomeningeal spread of low-grade tumors is rare. A rare cranial extension of brain leptomeningeal dissemination in an intramedullary pilocytic astrocytoma during adulthood is reported.

Case report. A 51 year-old-man with a recurrent intramedullary mass at $\mathrm{C} 5-\mathrm{C} 7$ level operated 4 times with all pathological anatomy reports describing the lesion as Pilocytic Astrocytoma developed, after 15 years from the diagnosis, visual hallucinations and his level of consciousness worsened to Glasgow coma score 13/15. The MRI showed highly enhanced cranial and spinal leptomeninges and paquimeninges with a micro-

Abreviaturas. DLM: diseminación leptomeníngea. DVP: derivación ventriculoperitoneal. EEG: electroencefalograma. GCS: escala de coma de Glasgow. LCR: líquido cefalorraquídeo. LI: tasa de titulación (labeling index). OMS: organización mundial de la salud. PA: astrocitoma pilocítico. PIC: presión intracraneal. PL: punción lumbar. RM: resonancia magnética. SNC: sistema nervioso central. TC: tomografía computarizada. 
nodular-granulomatous aspect associated with intense affectation of basal cisterns, subarachnoid spaces and convexity of both cerebral hemispheres suggestive of leptomeningeal spread of the spinal mass. The patient expired after three days.

Conclusion. Leptomeningeal spread is a rare phenomenon and when it happens usually doesn't change the primary tumor's behavior. In our case the aggressivenes could be explained by a potential malignization of the primary tumor that it wasn't documented because of the partial resectionss from the lasts surgeries or instead the tumor was actually a monomorphous pilomyxoid tumor.

KEY WORDS. Intramedullary spinal tumor. Leptomeningeal dissemination. Low-grade glioma. Pilocytic astrocytoma.

\section{Introducción}

Los tumores intramedulares representan entre el 5 y el $8 \%$ de todas las neoplasias espinales (epidurales $55 \%$; intradurales extramedulares $40 \%)^{22}$. Los astrocitomas son los segundos tumores más prevalentes a nivel intramedular en el adulto (alcanzando entre 24 y 30\%) tras los ependimomas (representando el 60\%). Pero son sólo el 1\% de todos los tumores del sistema nervioso central $(\mathrm{SNC})^{19}$. Se desarrollan principalmente en pacientes jóvenes (edad media 29 años) con una tendencia al predominio masculino $(63 \%)$ y representan el tumor intramedular más frecuente en la infancia (alcanzando el 90\%) ${ }^{24}$. Se identifican por ser tumores quísticos de bordes bien definidos y de crecimiento lento. Histológicamente se caracterizan por un patrón bifásico con proporciones variables de células bipolares con fibras de Rosenthal y células multipolares de textura laxa con microquistes y cuerpos granulares ${ }^{15}$. Es un tumor de línea media por lo que, en orden de frecuencia, su localización más habitual es: nervio óptico, quiasma óptico más hipotálamo, tálamo, ganglios basales, hemisferios cerebrales, cerebelo, tronco encefálico y médula espinal (es la menos frecuente pero no rara $)^{15}$. El 3\% de los astrocitomas se encuentran en la médula espinal ${ }^{24}$. Es raro encontrar diseminación de células tumorales a través del recorrido del líquido cefalorraquídeo (LCR) y esto sucede predominantemente en los meduloblastomas, ependimoblastomas, neuroblastomas centrales, ependimomas, tumores de células germinales y gliomas de alto grado ${ }^{19}$. La incidencia de la diseminación leptomeníngea (DLM) de los tumores de bajo grado se estima en un $5 \%$ en la presentación y entre un 7 y $10 \%$ con la progresión de la enfermedad ${ }^{1,22,23}$. La mayoría de las metástasis secundarias a gliomas medulares publicadas proceden de astrocitomas anaplásicos o glioblastomas multiformes y relativamente pocos de gliomas de bajo grado ${ }^{1,13,19}$. El glioblastoma multiforme representa el $80 \%$ de los gliomas

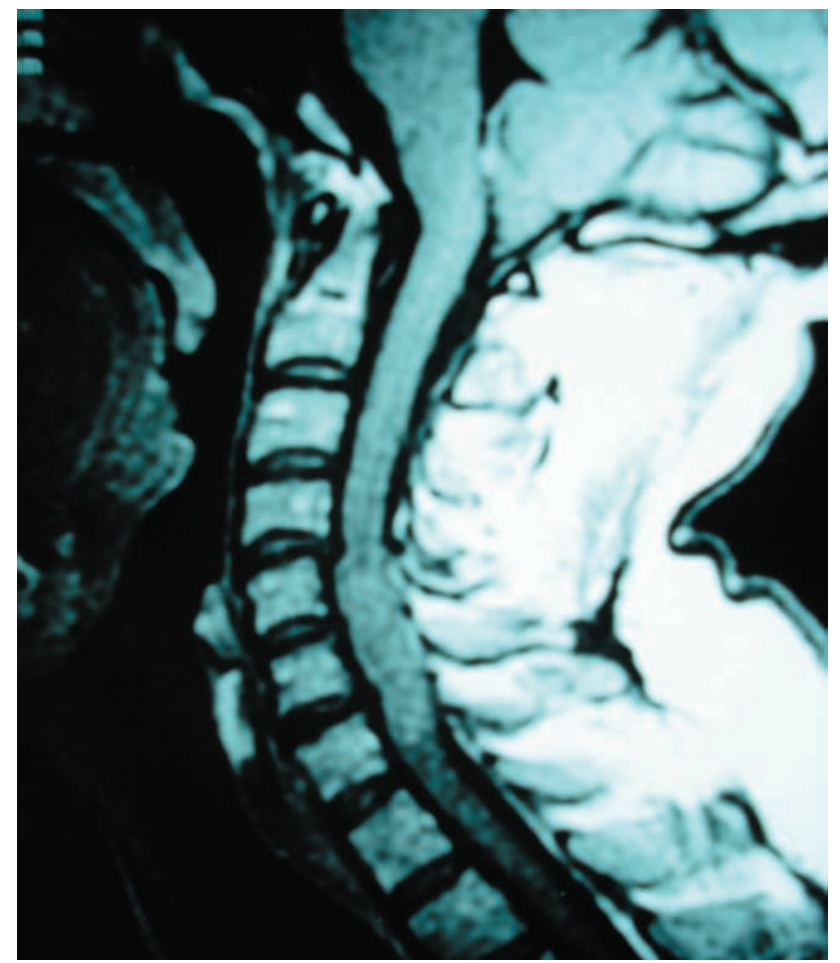

Figura 1. RM cervical corte sagital en secuencia T1 mostrando lesión prequirúrgica intramedular a nivel C5-C7.

medulares que dan metástasis cerebrales ${ }^{1}$.

\section{Caso clínico}

Publicamos un caso clínico de un varón de 51 años seguido por el Servicio de Neurocirugía tras ser operado en octubre de 1992, febrero de 1998, octubre de 1999 y enero 2007 por una tumoración intradural e intramedular a nivel de C5-C7 (figura 1). En todos los procedimientos quirúrgicos se utilizó aspirador ultrasónico y se realizó monitorización mediante registro de potenciales evocados somatosensoriales y motores. El grado de resección tumoral fue completo en la primera intervención quirúrgica y subtotal en el resto, dejando como volumen residual aproximadamente el 15\%. En todas las cirugías se usó tissucol durante el cierre. La $4^{\mathrm{a}}$ cirugía se realizó con intención paliativa.

En ninguno de los controles radiológicos realizados mediante RM cervical con espectroscopia se demostraron datos radiológicos que hiciesen sospechar malignización de la lesión. En dichas pruebas de imagen se mostró lesión ocupante de espacio intramedular a nivel C5-C6 isointensa respecto al parénquima medular circundante tanto en T1 como en T2 condicionando deformidad medular a dicho nivel.

Los diferentes informes de anatomía patológica describían la lesión como proliferación de células gliales 


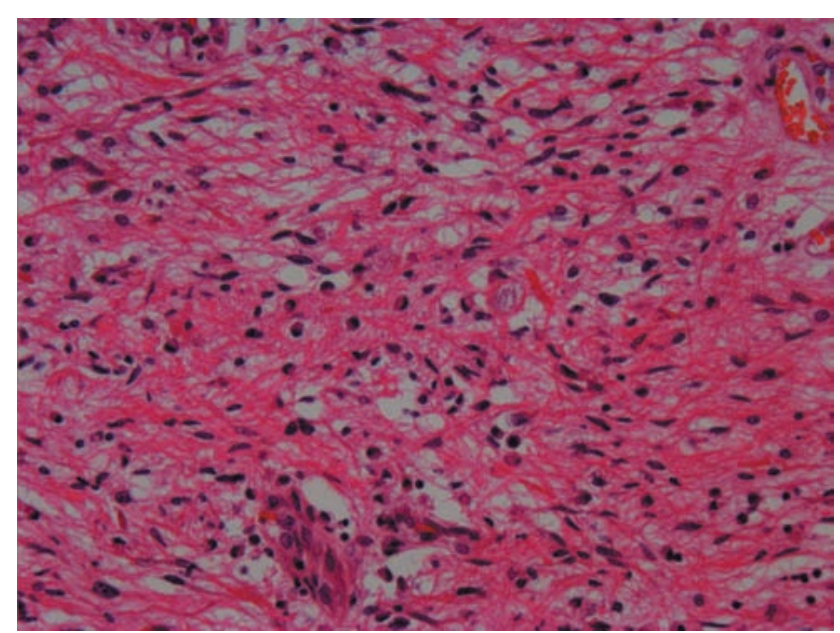

Figura 2. Preparación histológica de la pieza quirúrgica tumoral mostrando tejido teñido con hematoxilina $y$ eosina (20x) evidenciando núcleos fusiformes con ligero pleomorfismo e inclusiones intranucleares característicos de PA.

con núcleo ovoideo hipercromático y leve anisocariosis, observándose frecuentes fibras de Rosenthal en el estroma y ocasionales células de hábito gemistocítico (figura 2). Todas las muestras obtenidas en las diferentes cirugías se definieron como astrocitoma pilocítico (PA) con escasa actividad proliferativa evidenciada por la tasa de titulación de MIB-1 con Ki $67<1 \%$. En ningunas de las muestras obtenidas a lo largo de las diferentes cirugías se observó cambio en el grado de la histología que justificase una transformación anaplásica del astrocitoma así como tampoco se evidenció matriz mucosa ni estructura angiocéntrica que hiciesen sospechar la posibilidad de un astrocitoma pilomixoide. Tras la segunda cirugía (1998) se administró tratamiento radioterápico local con 50 Gy a nivel de C3-T3 (40 Gy iniciales seguidos de 10 Gy divididos en 2 Gy/semana durante 5 semanas). En las muestras histopatológicas obtenidas en

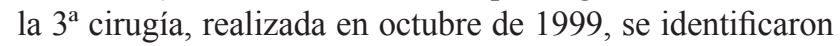
fragmentos de tejido hipocelular hialino con múltiples fragmentos de una proliferación paucicelular en abundante estroma fibroso con numerosas fibras de Rosenthal, junto a vasos de paredes hialinas. Las células constituyentes tenían núcleos fusiformes o redondeados con escaso pleomorfismo y ocasionales inclusiones intranucleares. Estas zonas mostraron muy leve actividad proliferativa mediante inmunohistoquímica para Ki-67 (inferior al 1\% de los núcleos de las células tumorales).

Antes de la última cirugía el paciente se encontraba con paraplejia y paresia de ambas manos con movimientos limitados a silla de ruedas. A los 2 meses de la $4^{\mathrm{a}}$ cirugía (marzo de 2007) desarrolló un pseudomeningocele cervicodorsal a tensión. Se realizó punción lumbar $(\mathrm{PL})$, sin alteraciones en la bioquímica, microbiología ni citología del LCR. Se resolvió parcialmente tras derivación cistoperitoneal. Ocho meses más tarde de la $4^{\mathrm{a}}$ intervención quirúrgica (septiembre de 2007) presentó episodios de desconexión del medio con alucinaciones visuales y olfativas acompañadas de cefaleas con pérdida progresiva de visión. Se observó de nuevo una gran colección líquida subcutánea bajo la cicatriz quirúrgica, blanda, fluctuante y sin signos de infección. Se realizó TC de cráneo urgente para poder realizar PL ante la sospecha de meningitis, evidenciando discreta dilatación ventricular. Se realizó punción lumbar midiendo una presión intracraneal (PIC) $>40 \mathrm{cmH} 20$ con hiperproteinorraquia sin otros cambios en la bioquímica, microbiología ni citología del LCR respecto a estudios previos, destacando la negatividad en la citología. Se descartó meningitis y se atribuyó el estado clínico y neurológico a hidrocefalia extracraneal secundaria a trastorno arreabsortivo por múltiples intervenciones e hiperproteinorraquia . En ningún momento se sospechó diseminación leptomeníngea debido a que los PA en el adulto no suelen desarrollar este fenómeno. Se colocó una derivación ventriculoperitoneal (DVP) con reservorio Pudenz de presión media. Durante el postoperatorio inmediato mostró clara mejoría neurológica volviendo a hablar y recuperar la escala de coma de Glasgow (GCS) 15/15 con resolución de los episodios de desconexión, alucinaciones y alteraciones visuales. Se resolvió también la tensión del pseudomeningocele pero no se reabsorbió la totalidad de su volumen. Tras 2 días de mejoría transitoria presentó nuevo deterioro progresivo consistente en tendencia al sueño y vómitos sin afectación de pares craneales ni nuevos déficits neurológicos. En la TC craneal urgente no se objetivaron cambios respecto a estudios previos, con tamaño ventricular dentro de la normalidad y con correcta colocación del catéter ventricular, sin complicaciones asociadas. Se realizó nueva PL confirmando PIC elevada con 27 cmH20 y sin signos de infección. La citología del LCR fue de nuevo negativa. El reservorio de la DVP fue reemplazado por uno de presión baja. Tras la cirugía el paciente mejoró nuevamente volviendo a su estado neurológico previo a los episodios relacionados con la hidrocefalia y el pseudomeningocele (estado cognitivo normal). En estudio mediante electroencefalograma (EEG) se identificó un patrón inespecífico con afectación global de la actividad bioeléctrica cerebral sugestiva de signos de sufrimiento cerebral difuso. En ningún momento se desarrolló fístula de LCR ni hubo problemas con las heridas. No se consideró tratamiento quirúrgico del pseudomeningocele para evitar reabrir una vez más la herida cervical.

A los 11 meses tras la $4^{\text {a }}$ cirugía (diciembre 2007), el paciente presentó de nuevo alucinaciones visuales y su nivel de conciencia se deterioró con una puntuación GCS 13/15 (O3, V4, M6). En la nueva RM craneal se evidenció un importante realce de las leptomeninges y paquimenin- 


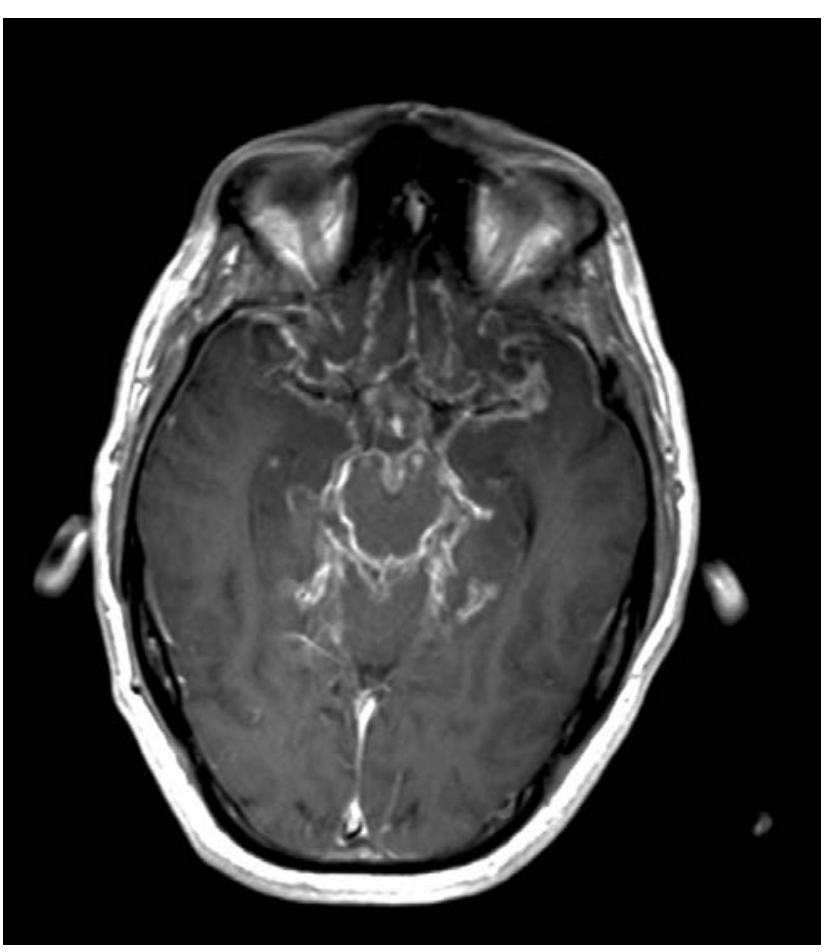

Figura 3. RM cervical corte axial en secuencia de densidad protónica mostrando realce de las leptomeninges y paquimeninges tras administración de gadolinio con aspecto micronodular-granulomatoso e intensa afectación de las cisternas basales, espacio subaracnoideo y ambos hemisferios cerebrales.

ges craneales y medulares, con aspecto micronodulargranulomatoso e intensa afectación de las cisternas basales, espacio subaracnoideo y ambos hemisferios cerebrales sugerente de DLM de la masa medular (figura 3). Tras repetir la PL, la citología del LCR siguió siendo negativa. En ese momento no se planteó biopsia debido a que la $4^{\mathrm{a}}$ cirugía se realizó con intención paliativa, el paciente había sufrido múltiples ingresos por complicaciones asociadas, su calidad de vida se había deteriorado más y la familia rechazaba más actitudes agresivas. No se realizaron estudios para descartar la posibilidad de carcinomatosis meníngea secundaria a una neoplasia externa al sistema nervioso central de carácter más agresivo por las mismas razones. Además, su diagnóstico habría cambiado poco el manejo clínico y su esperanza de vida, sin olvidar que la probabilidad de desarrollar carcinomatosis meníngea de un tumor previamente no diagnosticado es infrecuente $e^{2,8,26}$. El paciente falleció a los 3 días. No se realizó necropsia tras el fallecimiento debido a que la familia así expresó su deseo.

\section{Discusión}

En 1906, Grund describió por primera vez un tumor intramedular con extensión al espacio subaracnoideo 9 . En 1987, Johnson y Schwarz proporcionaron la primera documentación mediante tomografía computarizada de una DLM de un glioma de alto grado intramedular ${ }^{13}$. Muchos de los astrocitomas intramedulares con DLM tienen su tumor primario a nivel cervico-torácico ${ }^{11,22}$. Las áreas más comunes para la implantación de la DLM son las cisternas basales, las cisuras de Silvio y la cauda equina, (probablemente por la gravedad y el bajo flujo de LCR en estas áreas) ${ }^{1}$. Se ha propuesto una relación entre el lugar primario y la DLM. Los PA que surgen próximos a los ventrículos, cisternas y espacio leptomeníngeo en general, como la región quiasmática-hipotalámica y el vermis parecen tener una mayor tendencia para la diseminación a distancia ${ }^{22,23}$. En un estudio del St Jude Research Hospital la odds ratio de diseminación es 23 veces mayor para los PA localizados en la región hipotálamo-quiasmática que para los localizados en otras áreas ${ }^{23}$. De acuerdo con esta teoría, hay artículos que describen que los PA del nervio óptico y del quiasma difieren de algún modo en su patrón de crecimiento macroscópico y microscópico, con límites menos definidos y, por ello, difíciles de estadiar ${ }^{15}$. Por ello, el perfil clásico de un PA con DLM es el de un joven, frecuentemente un chico, con un gran tumor primario en la región hipotálamo-quiasmática y que posiblemente sufra de un síndrome diencefálico ${ }^{21}$. Siguiendo esta tendencia, hay publicaciones que describen gliomas de bajo grado medulares asociados a DLM que suceden en niños "mayores", representando una potencial nueva entidad clínica e histopatológica de un tumor de crecimiento lento mixto glioneuronal ${ }^{22}$. De acuerdo a ello, Tihan describe un subconjunto de gliomas de bajo grado con mayor tendencia a desarrollar diseminación respecto a los PA, al que llama "tumor monomorfo pilomixoide"27. Esta relativa nueva entidad, reconocida y aceptada por el grupo de trabajo de tumores cerebrales de la OMS, se ha descrito inicialmente en la región hipotálamo-quiasmática, aunque se han publicado artículos con localización a nivel intramedular ${ }^{14,19}$. Se ha publicado una mayor tasa de titulación (labeling index) (LI) de MIB-1 Ki67 en tumores pilomixoides monomorfos en relación a la normalmente descrita en los $\mathrm{PA}^{22,27}$.

En las primeras teorías se especulaba que la cirugía, y por tanto la manipulación quirúrgica, podrían contribuir a la diseminación a través de la exposición de células tumorales al LCR ${ }^{1}$. Posteriormente, la manipulación quirúrgica fue relegada a posible factor de riesgo para la DLM debido a que varios estudios han fracasado en demostrar correlación entre diseminación y resección ${ }^{1,3,18}$. En todos los casos publicados de PA con DLM la manipulación quirúrgica del tumor medular ha precedido a la aparición de DLM a nivel cerebral. Asimismo, la manipulación quirúrgica no es el único factor implicado; ya que hay un gran número de artículos que describen DLM en el momento del 


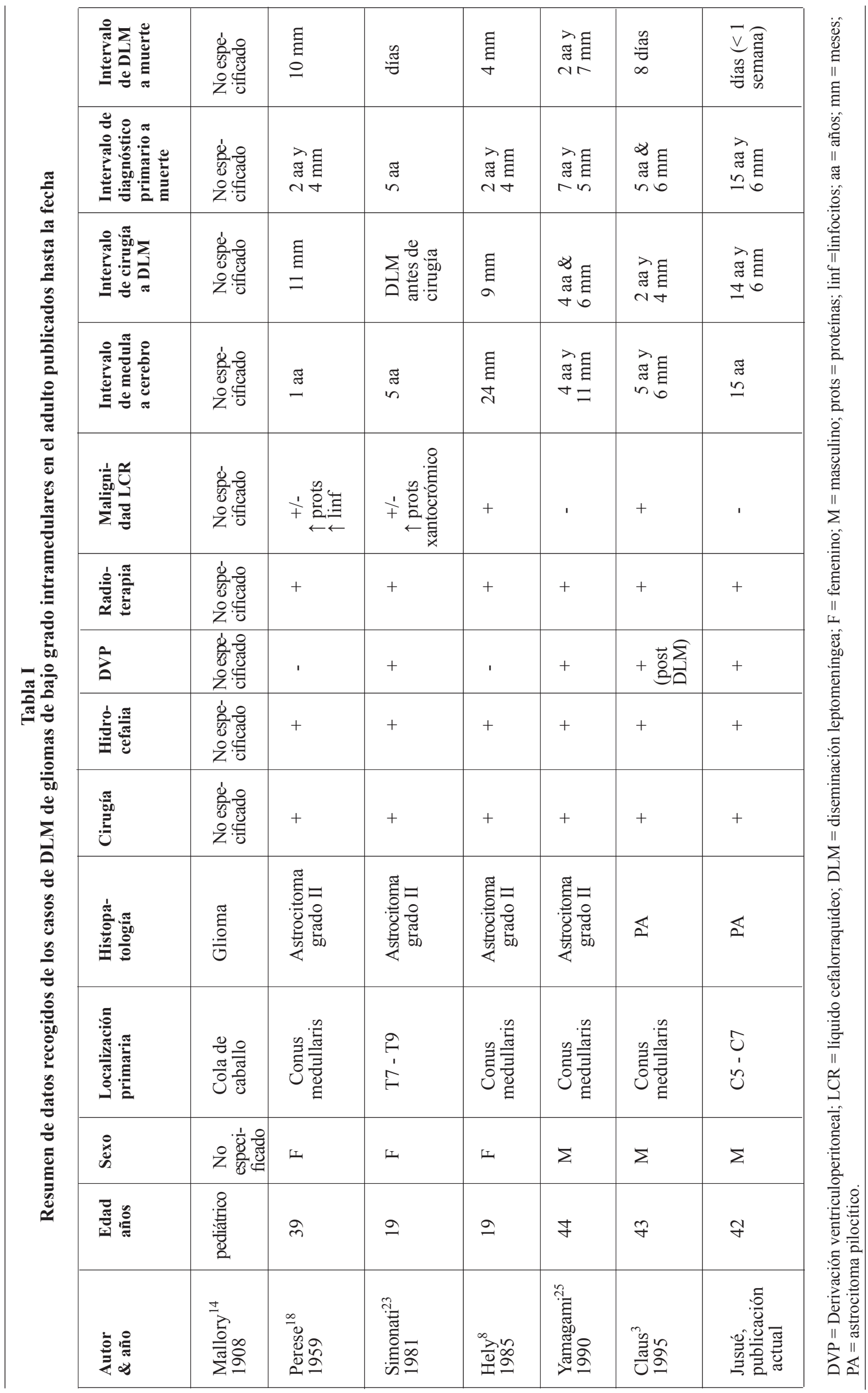


Tabla II

Media y rango de los intervalos de progresión de la tabla I

\begin{tabular}{|c|c|c|c|c|}
\hline & $\begin{array}{c}\text { Intervalo de médula } \\
\text { a cerebro }\end{array}$ & $\begin{array}{c}\text { Intervalo de cirugía } \\
\text { a DLM }\end{array}$ & $\begin{array}{c}\text { Intervalo de diagnóstico } \\
\text { primario a muerte }\end{array}$ & $\begin{array}{c}\text { Intervalo de DLM } \\
\text { muerte }\end{array}$ \\
\hline Media & 5 aa y $7 \mathrm{~mm}$ & 3 aa y $10 \mathrm{~mm}$ & 6 aa y $4 \mathrm{~mm}$ & $7 \mathrm{~mm}$ \\
\hline Rango & $(1 \mathrm{aa}-15 \mathrm{aa})$ & $(0-14$ aa y $6 \mathrm{~mm})$ & (2 aa y $4 \mathrm{~mm}-15$ aa y $6 \mathrm{~mm})$ & $($ días -2 aa y $7 \mathrm{~mm})$ \\
\hline
\end{tabular}

$\mathrm{DLM}=$ diseminación leptomeníngea; $\mathrm{aa}=\mathrm{años} ; \mathrm{mm}=$ meses .

diagnóstico ${ }^{1,5,17,25}$. La relación entre la positividad del LCR para células tumorales en gliomas de bajo grado y DLM permanece desconocida (tablas I y II).

Algunos autores han sugerido que el comportamiento clínico de los PA está relacionado con el $\mathrm{LI}^{7}$. Por otro lado, la tendencia de algunos PA para diseminar no ha sido firmemente correlacionada con una mayor tasa de proliferación o con ninguna otra característica histológica o genética conocida $^{22}$. Perilongo afirma que la diseminación de los gliomas de bajo grado parece proceder exclusivamente del espacio subaracnoideo o de la superficie ependimal, ya que las metástasis intraparenquimatosas en gliomas de bajo grado no se han publicado hasta ahora ${ }^{22}$. Esto no es una expresión de comportamiento maligno o de un futuro crecimiento agresivo ya que tanto la lesión primaria como los implantes suelen crecer lentamente y no están habitualmente asociados con transformación maligna ${ }^{15,17}$. De hecho, los implantes pueden ser asintomáticos y son posibles largas supervivencias incluso sin tratamiento adyuvante ${ }^{15}$. Revisando los casos de DLM de PA medulares de adultos publicados en la literatura, hemos encontrado una supervivencia media desde el establecimiento del diagnóstico de la DLM de aproximadamente 7 meses (rango 5 días a 31 meses). Nuestro caso representa el periodo más largo para el desarrollo DLM, sin embargo, cuando la DLM se establece el fallecimiento acontece más rápido que la media publicada hasta la fecha. Sólo uno de ellos ha mostrado DLM previo a la cirugía ${ }^{25}$. Actualmente, el comportamiento atípico de los PA no puede ser precedido ${ }^{4,10,20,25,28}$. Ya que los PA se caracterizan por el hecho de que su trasformación maligna sigue siendo un suceso raro; y éste se asocia a radioterapia previa. Los efectos mencionados anteriormente podrían haber ocurrido en el caso que publicamos en el presente artículo. Aunque es conocido que la trasformación maligna tras radioterapia muestra alta actividad mitótica y necrosis en empalizada, este fenómeno no se evidenció en las muestras histopatológicas ${ }^{7}$ de nuestras cirugías. En nuestro caso no fue demostrado un cambio ni en la histología (muestras quirúrgicas) ni en el LCR.
Como la heterogeneidad regional es una característica común en todos los gliomas, el tamaño de la biopsia y la extensión con la que representa al tumor afecta a la probabilidad de encontrar imágenes de mitosis $^{5-7}$. Las técnicas histológicas comunes no son suficientes para predecir el desarrollo de diseminación en los gliomas de bajo grado. En el momento actual los marcadores biológicos se encuentran en el horizonte de la futura predicción del comportamiento clínico de estas lesiones.

\section{Conclusiones}

La incidencia de diseminación leptomeníngea de gliomas de bajo grado es rara. En el adulto este fenómeno es aún más raro. En la edad pediátrica el desarrollo de DLM no suele cambiar el comportamiento del tumor primario. Sin embargo la tendencia evidenciada hasta la fecha en los casos adultos es hacia una aceleración de la agresividad. Tenemos 2 hipótesis para la rara progresión de nuestro paciente. Primera, podría haber ocurrido una transformación maligna del tumor intramedular, posiblemente inducida por radioterapia, la cual podría no haber sido evidenciada en la histología debido a la resección parcial en las últimas tres cirugías. Segunda, el tumor podría haber sido un tumor pilomixoide monomorfo no diagnosticado debido a muestras histológicas insuficientes.

\section{Bibliografía}

1. Abel, T.J., Chowdhary, A., Thapa, M., Rutledge, J.C., Geyer, J.R., Ojemann, J.: Spinal cord pilocytic astrocytoma with leptomeningeal dissemination to the brain. Case report and review of the literature. J Neurosurg. 2006; 105 (Suppl): 508-514.

2. Baiges-Octavio, J.J., Huerta-Villanueva, M.: Meningeal carcinomatosis. Rev Neurol. 2000; 31: 1237-1241.

3. Bryan, P.: CSF seeding of intra-cranial tumours: a study of 96 cases. Clin Radiol. 1974; 25: 355-360.

4. Claus, D., Sieber, E., Engelhardt, A., Rechlin, T., Neu- 
bauer, U., Volk, B.: Ascending central nervous spreading of a spinal astrocytoma. J Neurooncol. 1995; 25: 245-250.

5. Coons, S.W., Johnson, P.C.: Regional heterogeneity in the DNA content of human gliomas. Cancer. 1993; 72): 30523060 .

6. Coons, S.W., Johnson, P.C.: Regional heterogeneity in the proliferative activity of human gliomas as measured by the Ki-67 labeling index. J Neuropathol Exp Neurol. 1993; 52: 609-618.

7. Giannini, C., Scheithauer, B.W., Burger, P.C., Christensen, M.R., Wollan, P.C., Sebo, T.J.: Cellular proliferation in pilocytic and diffuse astrocytomas. J Neuropathol Exp Neurol. 1999; 58: 46-53.

8. Groves, M.D.: New strategies in the management of leptomeningeal metastases. Arch Neuro. 2010; 67: 305-312.

9. Grund, G.: Uber die diffuse Ausbreitung von malignen Tumoren, insbesondere Gliosarkoma in den Leptomeningen. Dtsch Nervenheilk. 1906; 31: 283-312.

10. Hely, M., Fryer, J., Selby, G.: Intramedullary spinal cord glioma with intracranial seeding. J Neurol Neurosurg Psychiatry. 1985; 48: 302-309.

11. Houten, J.K., Cooper, P.R.: Spinal cord astrocytomas: presentation, management and outcome. J Neurooncol. 2000; 47: 219-224.

12. Huang, T., Zimmerman, R.A., Perilongo, G., Kaufman, B.A., Holden, K.R., Carollo, C.: An unusual cystic appearance of disseminated low-grade gliomas. Neuroradiology. 2001; 43): 868-874.

13. Johnson, D.L., Schwarz, S.: Intracranial metastases from malignant spinal-cord astrocytoma. Case report. J Neurosurg. 1987; 66: 621-625.

14. Komotar, R.J., Carson, B.S., Rao, C., Chaffee, S., Goldthwaite, P.T., Tihan, T.: Pilomyxoid astrocytoma of the spinal cord: report of three cases. Neurosurgery. 2005; 56: 191.

15. Louis, D.N.M., Ohgaki, H., Wiestler, O.D., Cavenee, W.K.: Who Classification of Tumours of the Central Nervous System. $4^{\circ}$ ed. IARC; 2007. pp 14-21

16. Mallory, F.: The results of the application of special histological methods with the study of tumors. J exp Med. 1908; 10: 575-593.

17. Ng, H.K., Leung, C.H., Boet, R., Poon, W.S.: Spinal cord pilocytic astrocytoma with cranial meningeal metastases. J Clin Neurosci. 2001; 8: 374-377.

18. Obana, W.G., Cogen, P.H., Davis, R.L., Edwards, M.S.: Metastatic juvenile pilocytic astrocytoma. Case report. J Neurosurg. 1991; 75: 972-975.

19. Peraud, A., Herms, J., Schlegel, J., Müller, P., Kretzs- chmar, H., Tonn, J.: Recurrent spinal cord astrocytoma with intraventricular seeding. Childs Nerv Syst. 2004; 20: 114118.

20. Perese, D.M., Slepian, A., Nigogosyan, G.: Postoperative dissemination of astrocytoma of the spinal cord along the ventricles of the brain; a case report. J Neurosurg. 1959; 16: 114-119.

21. Perilongo, G., Carollo, C., Salviati, L., Murgia, A., Pillon, M., Basso, G.: Diencephalic syndrome and disseminated juvenile pilocytic astrocytomas of the hypothalamic-optic chiasm region. Cancer. 1997 1; 80: 142-146.

22. Perilongo, G., Garrè, M.L., Giangaspero, F.: Lowgrade gliomas and leptomeningeal dissemination: a poorly understood phenomenon. Childs Nerv Syst. 2003; 19: 197203.

23. Pollack, I.F., Hurtt, M., Pang, D., Albright, A.L.: Dissemination of low grade intracranial astrocytomas in children. Cancer. 1994 1; 73: 2869-2878.

24. Roonprapunt, C., Houten, J.K.: Spinal cord astrocytomas: presentation, management, aTJ, nd outcome. Neurosurg Clin N Am. 2006; 17: 29-36.

25. Simonati, A., Mazza, C., Rizzuto, N.: An unusual case of meningeal gliomatosis. Acta Neuropathol Suppl. 1981; 797-800.

26. Taillibert, S., Laigle-Donadey, F., Chodkiewicz, C., Sanson, M., Hoang-Xuan, K., Delattre, J.Y.: Leptomeningeal metastases from solid malignancy: a review. J Neurooncol. 2005; 75: 85-99.

27. Tihan, T., Fisher, P.G., Kepner, J.L., Godfraind, C., McComb, R.D., Goldthwaite, P.T.: Pediatric astrocytomas with monomorphous pilomyxoid features and a less favorable outcome. J Neuropathol Exp Neurol. 1999; 58: 1061-1068.

28. Yamagami, T., Kikuchi, H., Higashi, K., Goto, Y., Imataka, K.: Intracranial metastasis of a spinal cord astrocytoma-case report. Neurol Med Chir (Tokyo). 1990; 30: 69-73.

Jusué-Torres, I.; Alcázar-Vaquerizo, L.; Gómez-Angulo, J.C.; Navarro-Torres, R.; López-Serrano, R.; GarcíaMiralles, N.: Diseminación leptomeníngea de un astrocitoma pilocítico cervical en el adulto: publicación de un caso y revisión de la literatura. Neurocirugía 2011; 22: 445-452.

Correspondencia: Jusué Torres, Ignacio. Servicio de Neurocirugía. Hospital Universitario de Getafe. Carretera de Toledo Km 12500. Getafe 28905 (Madrid) España

Mail: ijusue.hugf@salud.madrid.org 


\section{Comentario al trabajo Diseminación leptomeníngea de} un astrocitoma pilocítico cervical en el adulto: publicación de un caso y revisión de la literatura de I. Jusué y cols.

Los autores presentan un caso de astrocitoma pilocítico cervical recurrente en un adulto, que fue intervenido en múltiples ocasiones a lo largo de 15 años, además de tratado con radioterapia local y que finalmente experimentó diseminación a leptomeninges encefálicas, provocando un rápido deterioro neurológico del paciente y su fallecimiento. El diagnóstico de extensión craneal se sustenta únicamente en los estudios radiológicos practicados, ya que la citología de LCR fue negativa y una biopsia meníngea fue desestimada, al igual que rechazada la realización de la necropsia tras el fallecimiento, lo que constituye la principal limitación del trabajo.

La diseminación leptomeníngea de un astrocitoma medular puede considerarse un fenómeno muy poco común. Los factores que parecen influir de manera decisiva en la misma incluyen principalmente la proximidad del tumor a la superficie pial y la presencia de una histología más agresiva (astrocitomas anaplásicos y glioblastomas). Como elemento menos aceptado se discute la manipulación quirúrgica que podría facilitar la siembra de células neoplásicas a distancia.

Atendiendo a la propia circulación del LCR como vehículo de extensión neoplásica, las áreas más frecuentes de diseminación incluyen las cisternas basales, la cisura de Silvio y la cauda equina, tanto por el efecto gravitatorio como por el enlentecimiento de flujo en estas áreas. Sin embargo, el patrón de diseminación en este caso parece contrario a la propia hidrodinámica del LCR, ya que no se describe diseminación caudal a la lesión primaria y sí craneal.
2011; 22: 445-452

La diseminación leptomeníngea de un astrocitoma pilocítico ha sido descrita con mayor frecuencia en la variante pilomixoide. Este subtipo de astrocitoma, más común en la región hipotalámica, muestra características histológicas peculiares, cursa generalmente con clínica más agresiva y presenta mayor tendencia a la diseminación a través del LCR; sin embargo, esta posibilidad fue desestimada tras el análisis de las repetidas muestras histológica obtenidas en las múltiples intervenciones quirúrgicas, ya que tras varias resecciones parciales, el tumor presentó siempre similares características histológicas, descartándose también la transformación anaplásica, con la limitación que implica el hecho de tratarse de resecciones incompletas.

Es posible que en el caso descrito por Jusué y col., la larga evolución de la enfermedad, al no ser posible la resección tumoral completa, y las repetidas manipulaciones quirúrgicas practicadas, hayan podido contribuir a la diseminación del tumor, transformándose finalmente un proceso de evolución crónica clínicamente agresivo con rápido y fatídico desenlace en un breve periodo de tiempo.

Tal como manifiestan los autores, las técnicas histológicas habituales no parecen válidas para predecir la diseminación leptomeníngea de un astrocitoma por lo que es preciso definir otros marcadores biológicos que permitan anticipar esta conducta particularmente agresiva y observada incluso precozmente en algunos casos para determinar actuaciones terapéuticas más enérgicas en los pacientes de mayor riesgo.

J. Domínguez Báez Tenerife 